\title{
Modelling and Realization of Pneumatics based Wall Climbing Robot for Inspection Applications
}

\author{
G. Muthukumaran ${ }^{\# 1}$, U. Ramachandraiah ${ }^{\# 2}$ \\ \# Department of Electronics and Instrumentation Engineering, \\ Hindustan Institute of Technology and Science (Hindustan University), Chennai, India \\ gmkumaran@hindustanuniv.ac.in \\ 2uppur@hindustanuniv.ac.in
}

\begin{abstract}
Climbing robots are highly in need for catering the inspection of the high rise buildings. Climbing Robots has to work in challenging environments and has to maneuver vertically against gravity and orient towards the specified target by adapting to various surfaces. The proposed Wall Climbing Robot weighs $1 \mathrm{Kg}$, works with four active suction cups and driven with pneumatic supply cylinder drive assembly. The robot moves by absolute holding of two suction cups at any instance and remaining two cups are allowed free to enable forward movement of the robot. The robot consists of vacuum generator, suction cup, pneumatic driven traverse assembly, controller and wireless high resolution camera. The designed robot is highly suitable for the continuous monitoring and inspection of high rise buildings. The vacuum generator is externally positioned and suction is derived through pneumatic supply hose. The developed robot climbs on the vertical wall at a velocity of $1.5 \mathrm{~cm} / \mathrm{s}$.
\end{abstract}

Keyword -Wall climbing robot, suction cup, smooth surface, pneumatic operation, programmable logic controller

\section{INTRODUCTION}

The design of a wall climbing robot (WCR) requires appropriate/good adhesion mechanism so that the robot latches on to the wall without compromising with mobility. In this context many climbing robot designs are attempted by researchers by using suction cups. The required negative pressure to hold the robot is derived through either external vacuum generators or on board vacuum pumps. To check the defects in concrete walls, SaekoTokuomi et.al.developed suction cup based climbing robots. Two major problems faced are inadequate ring-seal decompression in suction cups and suction cup slippage [1]. To increase the reliability of the holding of the robot is attempted with multiple cups by providing multi-legged wall-climbing robot which latches to a wall with negative pressure adsorption [2]. Wall climbing robots for inspecting industry pipe network is developed with bio-mechanical suction cups actuated by shape memory alloy (SMA) actuators [3]. These robots can move either straight or can turn with a fixed angle on an inclined glass wall. Wall-climbing robots employing single suction cup are used in ultrasonic inspection of steel storage tanks and cleaning high-rise buildings. The robots have three major parts. A vacuum pump, a sealing mechanism and a driving mechanism [4]. The researchers developed adaptive trajectory tracking approach for WCR. The major challenge faced is to ensure complete contact of the drive wheels with the wall surface which affects robot movement. [5]. The adhering capability is one of the most important performance indices of WCRs. Another approach to achieve this is the sliding suction cup (SSCs) using fluid network theory [6]. H. Zhang et.al.developed Sky cleaner 3, which is used for wall cleaning, maintenance of high-rise buildings and glass-wall cleaning [7]. Critical suction, based on the negative suction and thrust force is analyzed for understanding the smart movement of the wall climbing robot reliably on the wall [8]. A miniature smart robotic foot, consisting of integrated vacuum pump, suction cup, pressure sensor, micro-valve, micro-controller, a video micro-camera, wireless interface and thin film battery was designed, fabricated and tested [9]. Tohru Miyake et.al. attempteda vacuum-based wet adhesion system for WCRs with adherability and low friction performance when a suction cup clings on rough surface (say, concrete wall) [10]. T. C. Apostolescuet.al. used electro-pneumatic vacuum cups to address several factors which effect the robot in holding, moving on horizontal and vertical surfaces [11]. Z. Wang et.al presented Walkman-I (a new kind of flexible pneumatic wall-climbing robot) composed of a flexible pneumatic actuator, a flexible pneumatic spherical joint and six suction cups [12]. The alternative techniques by using low-frequency vibrating passive attachment principle to hold the robot is also suggested [13]. J. Zhu et.al. developed a service climbing robot (with visual sensing mechanism) so as to clean the glass wall of high-rise buildings [14]. Though few climbing robots are realized with on board vacuum generator and dc motor driven drive assembly, the robot cannot be continuously operated due to high power requirements and limitations in payload capacity. This proposed robot is developed for vertical wall climbing applications, highly suitable for continuous monitoring and inspection. Hence vacuum is generated using an external Vacuum generator and robot movement is effected through pneumatic drive assembly. 


\section{WALL CLIMBING ROBOT - SYSTEM ARCHITECTURE}

The building blocks of the proposed climbing robot consists of electrical assembly, pneumatic assembly and mechanical assembly. The system block diagram is shown in Fig.1.

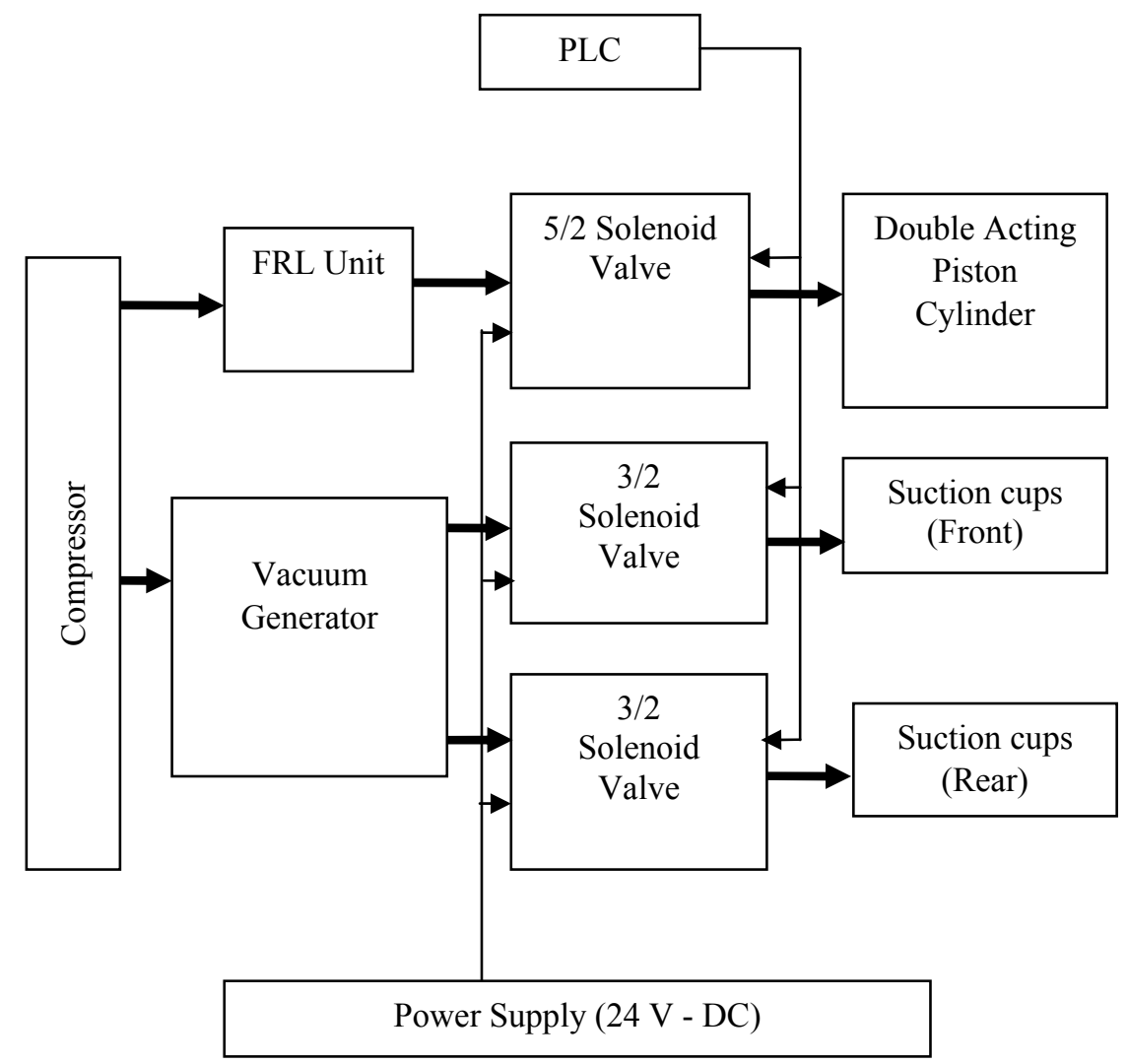

Fig. 1. System Block Diagram

\section{A. Electrical Module}

Fig. 2 explains the functional connection diagram of the proposed climbing robot.

1) Power supply: ZEN PLC, Vacuum Generator and Electro pneumatic valves requires $24 \mathrm{~V}$ DC.

2) Control Module: Programmable Logic Controller (PLC) (Zen make, Model no.10C1DR-D-V1) is programmed using ladder logic, which controls the sequence of switching. This PLC has six inputs and four outputs.

The four relay outputs are mentioned below:

- Q0 - Vacuum generator

- Q1, Q3 - 3/2 Valve - Vacuum switch for front and rear pair of legs

- Q2 - 5/2 Valve - Double acting piston cylinder

The robot attachment to the wall and traverse on the wall is controlled by the four output relays. 


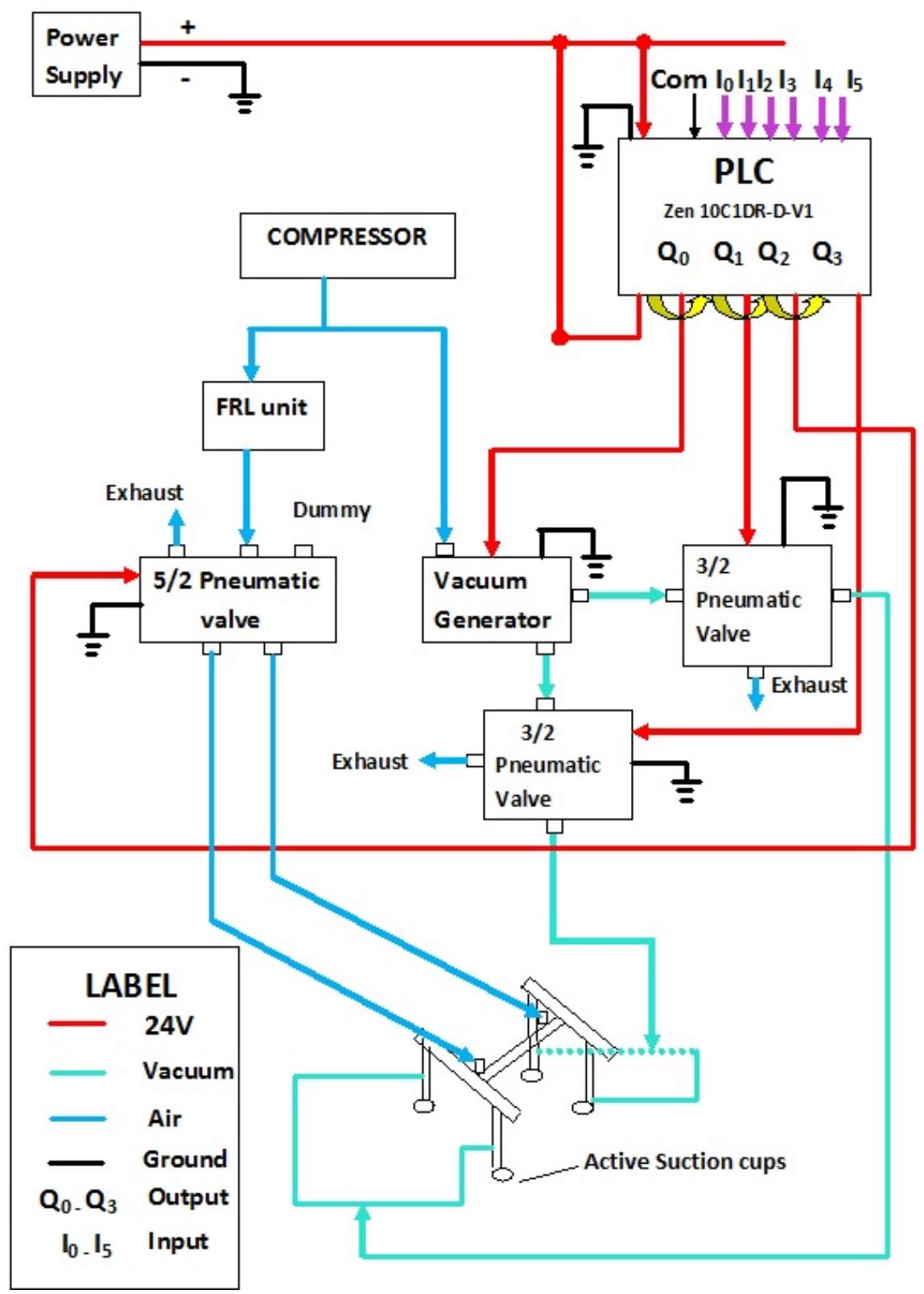

Fig. 2 Functional Connection Diagram

B. Pneumatic Assembly

The compressed air is used as a working medium. The vacuum generator generates vacuum using Bernoulli's principle. The air pressure is maintained, which supplies the pneumatic valves and the vacuum generator through FRL (filter regulator lubricator) unit. The air pressure $7 \mathrm{Kg} / \mathrm{cm}^{2}$ is given as input to the vacuum generator. The vacuum generator output is connected to the inputs of the $3 / 2$ normally open pneumatic valve. The front and rear pair of suction cups is connected to the outputs of the $3 / 2$ normally open pneumatic valve. The double acting piston is integrated in the mechanical assembly through $5 / 2$ normally open pneumatic valve to enable robot movement.

C. Mechanical Assembly

As shown in Fig. 3 the developed wall climbing robot comprises of Aluminum guide rod, four grippers using suction cups, double acting cylinder and balance support roller. The major specifications of the developed robot is listed in table 1 


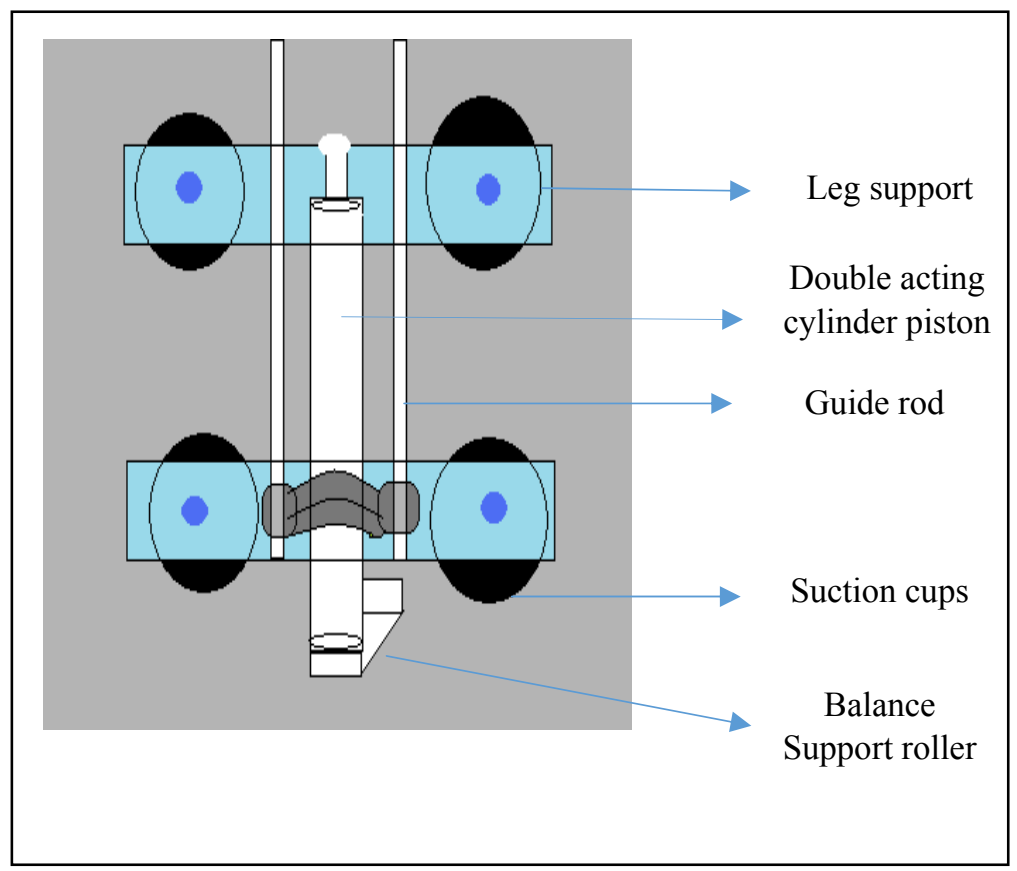

Fig. 3 Implementation Diagram

TABLE I Wall climbing robot Mechanical Specifications

\begin{tabular}{|l|c|}
\hline Mass & $1.0 \mathrm{Kg}$ \\
\hline Height from the base $h$ & $11 \mathrm{~cm}$ \\
\hline length from tail end to rear cup $L$ & $13.5 \mathrm{~cm}$ \\
\hline $\begin{array}{l}\text { Distance between the front and } \\
\text { rear cup ( Uncompressed state) } L_{u}\end{array}$ & $34.5 \mathrm{~cm}$ \\
\hline $\begin{array}{l}\text { Distance between the front and } \\
\text { rear cup ( Compressed state) } L_{c}\end{array}$ & $24.5 \mathrm{~cm}$ \\
\hline
\end{tabular}

III. MATHEMATICAL MODELLING

Notational convention:

$N_{f}$ - Reaction forces exerted by the front suction cup

$N_{r}$ - Reaction forces exerted by the rear suction cup

$f_{f}$ - Frictional forces between the front suction cup and the wall

$f_{r}$ - Frictional forces between the rear suction cup and the wall

$W_{-}$Weight of the robot

$L$ - Position of the rear suction cup from the bottom edge

$L_{c}$ - Spacing between the front and rear suction cups (compressed state)

$L_{u}$ - Spacing between the front and rear suction cups (uncompressed state)

$h$ - Distance from the wall to the centre of gravity

$F_{s}$ - Suction force of the suction cup

$\mu_{- \text {Static frictional coefficient }}$

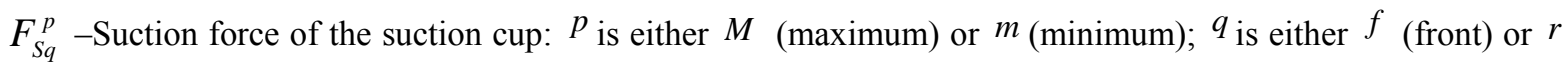
(rear)

Fig. 4 shows the force balance diagram of the proposed climbing robot. 


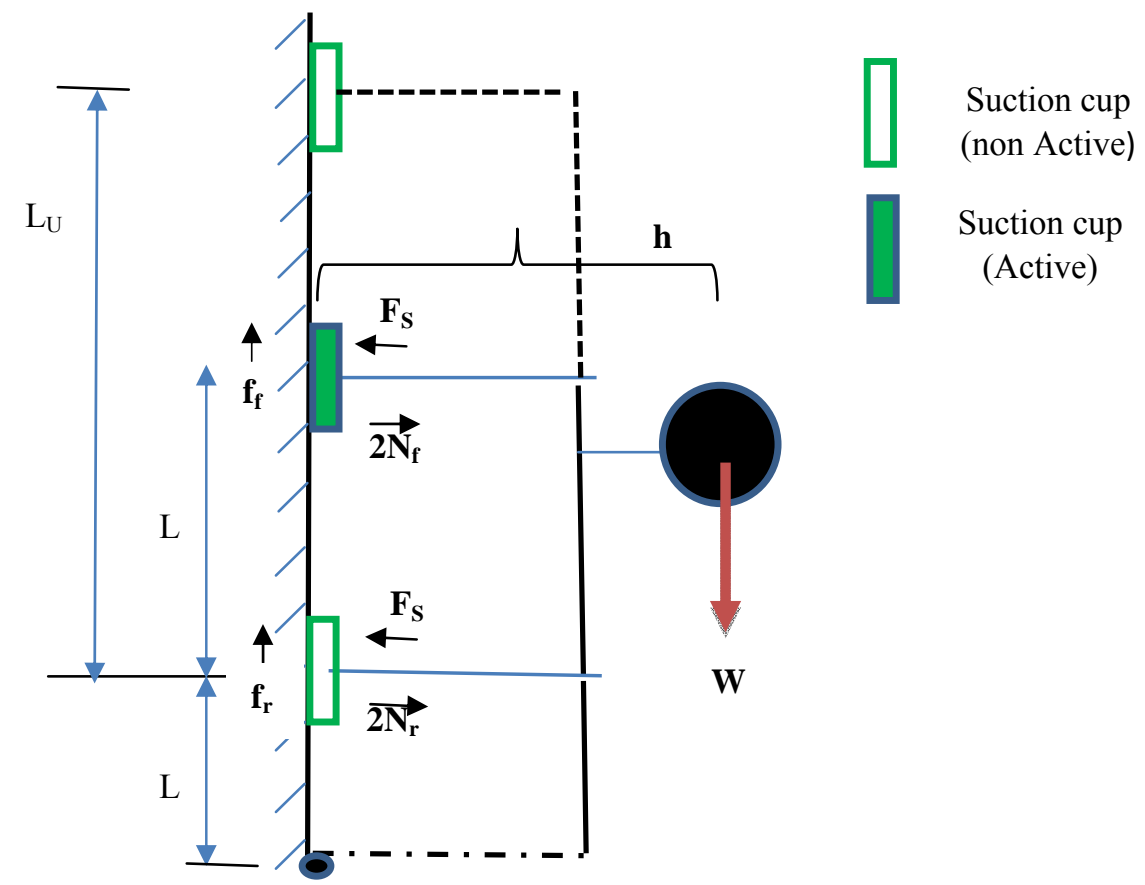

A

Fig. 4 Force Balance Diagram

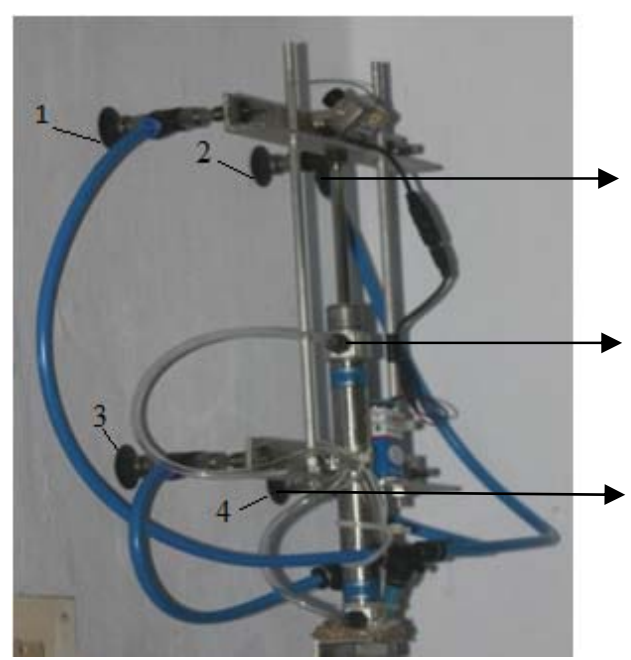

Suction cups 1, 2 fixed to the piston rod of the Double acting cylinder assembly

Double acting cylinder piston assembly

Suction cups 3, 4 fixed to the Double acting cylinder assembly

Considering the Force balance,

$\mathrm{X}$ axis:

$2 N_{f}+2 N_{r}-2 F_{s}=0$

Since the robot functions with two front suction cups and two rear suction cups, $2 N_{f}$ and $2 N_{r}$ are considered in (1). Since the robot movement is achieved by holding and release of either the two front or two rear suction cups at any instant of time, $2 F_{s}$ appears in (1).

Y axis:

$2 f_{f}+2 f_{r}-W=0$

As the robot climbs over a wall, the frictional force, coefficient of friction and the normal reaction are connected as

$\left.\begin{array}{l}f_{f} \leq \mu N_{f} \\ f_{r} \leq \mu N_{r}\end{array}\right\}$ 
$\Rightarrow f_{f}+f_{r} \leq \mu\left(N_{f}+N_{r}\right)$

Using (1), (2) and (3) results in

$W \leq 2 \mu F_{S}$

The movement of the robot is realized by using a double acting cylinder piston, where in the piston end holds the front suction cups 1,2 and the cylinder assembly houses the rear suction cups 3, 4 as shown in Fig.5. The rear suction cups 3, 4 holds and the front suctions cups 1, 2 are free for enabling the traverse of the robot. The sequence is repeated by alternatively holding and release of the front and rear suction cups.

Moment around the Point A:

Case 1(compressed mode): Suction cup 1, 2 not active.

Suction cup 3, 4 are in hold and active.

$2\left(F_{s r}^{m}-N_{r}\right) L=2 N_{f}\left(L+L_{c}\right)+W h$

Case 2 (Uncompressed mode): Suction cup 1, 2 not active.

Suction cup 3, 4 are in hold and active.

$2\left(F_{S r}^{M}-N_{r}\right) L \geq 2 N_{f}\left(L+L_{u}\right)+W h$

Obviously, from (5) and (6), it can be seen that $F_{S r}^{M}>F_{S r}^{m}$ since $L_{u}>L_{c}$

Case 3 (Compressed mode): Suction cup 1, 2 are in hold $\&$ active.

Suction cup 3, 4 is not active.

$2\left(F_{s f}^{m}-N_{f}\right)\left(L_{u}+L\right)=2 N_{r} L+W h$

Case 4 (Uncompressed mode): Suction cup 1, 2 are in hold \& active.

Suction cup 3, 4 is not active.

$2\left(F_{S r}^{M}-N_{f}\right)\left(L_{c}+L\right)=2 N_{r} L+W h$

Obviously, from (7) and (8), it can be seen that $F_{S f}^{M}>F_{S f}^{m}$ since $L_{u}>L_{c}$

Further, it is clear that, the following holds always:

$F_{S f}^{m}<F_{S r}^{m}$

$F_{S f}^{M}<F_{S r}^{M}$

Though the force needed in compressed mode is smaller than that in uncompressed mode, during the current work, same amount of force (slightly greater than what is needed in uncompressed form) is applied in both cases, which has to be optimized as future work.

\section{Theoretical Suction cup holding force calculation:}

Mass, $m=1 \mathrm{Kg}$

$L=13.5 \mathrm{~cm}$

$\mu=0.6$

$S=1.5$ (Safety factor)

Substituting in (4),

$F_{S} \quad=8.175 \mathrm{~N}$ (for a single suction cup)

The proposed robot holds with 2 suction cups at any instant, considering the safety factor the theoretical holding force of the robot will be

$$
F_{S} \quad=24.525 \mathrm{~N} \quad(2.5 \mathrm{Kgf})
$$

It is observed that from (4), for preventing the robot from sliding off from the wall each suction cup should have a holding force of $8.175 \mathrm{~N}$.

The condition for the robot not to fall off from the wall can be estimated through (5), (6).

Substituting the parameters of table 1 in (5), and assuming a fall off limit of zero reaction force on the suction cup , $N_{r}=0, N_{f}=F=0.5 \mathrm{~N}$, weight of the robot $\mathrm{m}=1 \mathrm{Kg}$, the minimum required holding force of each cup will be

$F_{S}=5.4 \mathrm{~N}$ (compressed mode): Suction cup 1, 2 not active, Suction cup 3, 4 are in hold and active.

Substituting the parameters of table 1 in (6), and assuming a fall off limit of zero reaction force on the suction cup, $N_{r}=0, N_{f}=F=0.5 \mathrm{~N}$, weight of the robot $\mathrm{m}=1 \mathrm{Kg}$, the minimum required holding force of each cup will be

$F_{S}=5.7 \mathrm{~N}$ (uncompressed mode): Suction cup 1, 2 not active, Suction cup 3, 4 are in hold and active. 


\section{SELECTION OF SUCTION CUP, VACUUM GENERATOR}

A. Selection of suction cup

The effective diameter of the suction cup determines its holding force. The required diameter $d$ of the each suction cup can be determined by

$d=1.12 \sqrt{\frac{m S}{P n \mu}}=1.54 \mathrm{~cm} \simeq 15 \mathrm{~mm}$

where number of the suction cups $n=2$, mass of the proposed robot $m=1 \mathrm{Kg}$, friction coefficient $\mu=0.6$ for dry surfaces, System vacuum $P=-0.6$ bar and a safety factor of $S=1.5$.

Based on (10), NPFYN20, model suction cup with diameter of $20 \mathrm{~mm}$ made of Acrylonitrile-Butadiene Rubber is selected for the proposed robot. [15]

B. Selection of Vacuum Generator

The vacuum generator capacity to empty the suction cup i.e., suction rate $V_{S}$ is governed by the suction cup diameter. The suction rate $V$ is given by,

$V=n V_{S}=16.61 / \mathrm{min}$

Where $V_{S}=8.3 \mathrm{l} / \mathrm{min}$ [15], number of the suction cups $n=2$

Hence from (11), NSCPI 15 Vacuum Generator capable of ejecting 75 1/min is selected[15].

\section{RESULTS AND DISCUSSION}

Fig.6 depicts the developed prototype wall climbing robot with inspection camera and accessories. The robot is tested in real time and capable to traverse without any slide or drop off .Though the weight of the robot proposed is limited to $1 \mathrm{Kg}$, it can be enhanced by increasing the suction cup diameter and suction rate of the vacuum generator. Fig.7 helps to identify the safe working region of the robot. The plot of Eq. (4) represents the robot in holding condition and not allow to slide. The plot of Eq. (5) represents the robot in about to fall off condition and helps to estimate the minimum holding force of the suction cup.
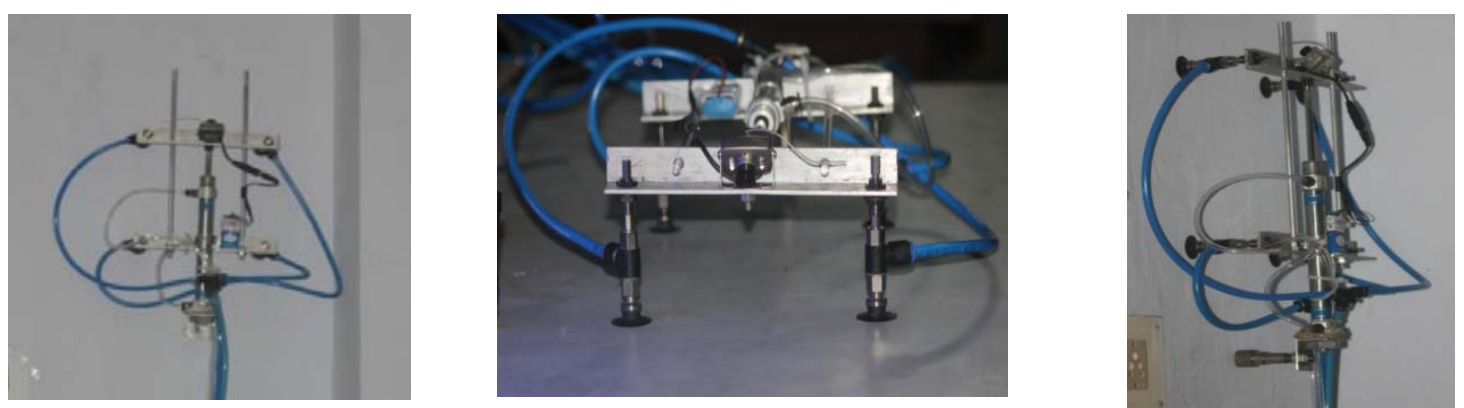

Fig- 6 Prototype Climbing robot 


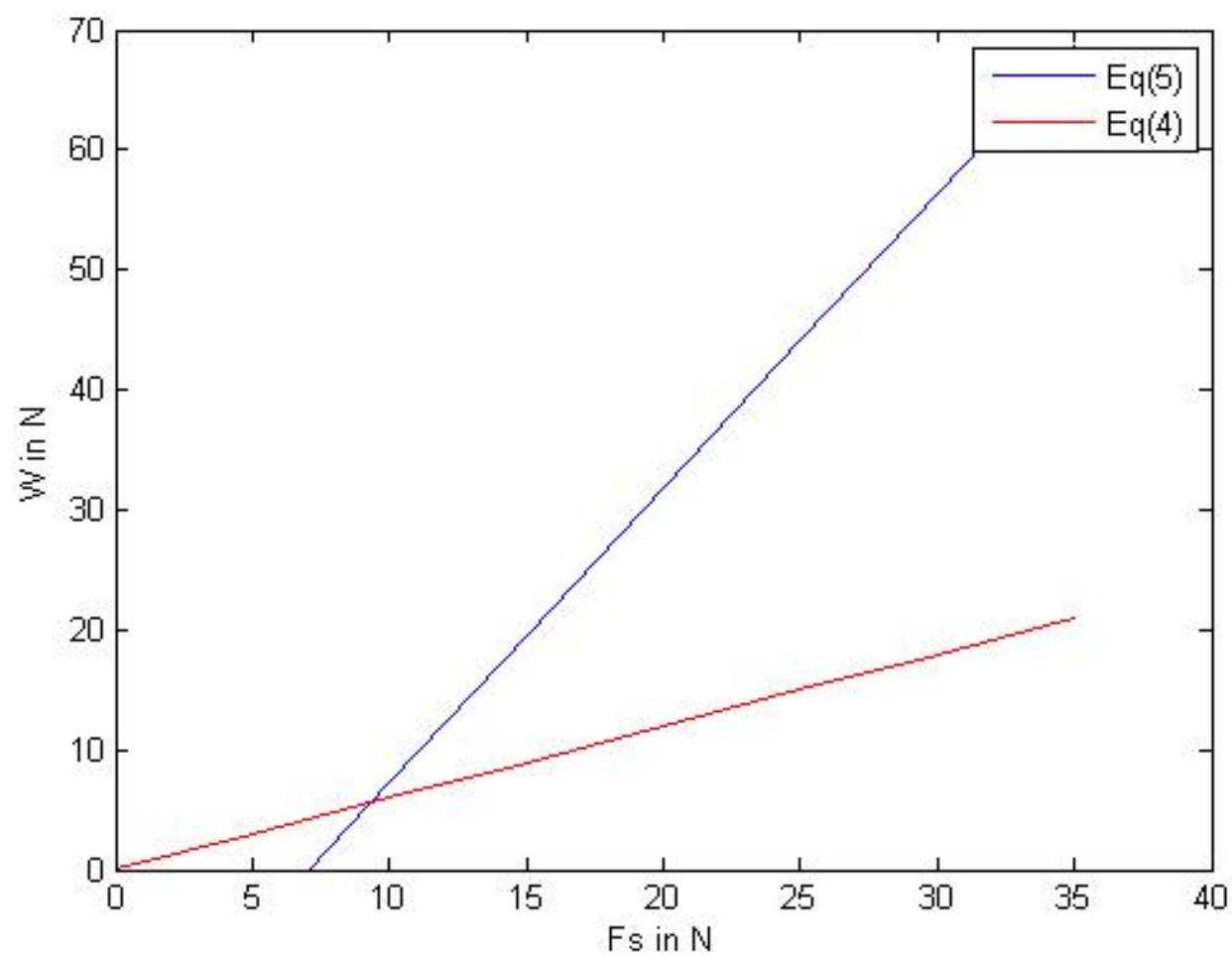

Fig- 7(compressed mode): Suction cup 1, 2 not active. Suction cup 3, 4 are in hold and active.

\section{CONCLUSION}

In this work the pneumatic based wall climbing robot is mathematically modelled and realized using suction cups, pneumatic driven traverse assembly, Programmable logic controller and wireless high resolution camera. This robot is designed for continuous monitoring and inspection applications of high rise buildings. It can be concluded that $F_{S}=5.4 \mathrm{~N}$, derived from Eq. (5) will be the minimum holding force for the single cup. Hence to operate the proposed robot in the safety region, a holding force for each cup is maintained at $F_{S}=8.175 \mathrm{~N}$. The robot is able to traverse at a speed of $1.5 \mathrm{~cm} / \mathrm{s}$. The limitations of the robot are single degree of traverse, slow travel time. This will be addressed in the future research and development of a wall climbing robot.

\section{REFERENCES}

[1] SaekoTokuomi and Kazuya Mori, "Suction Cup for Concrete Wall Testing Robot," Journal of Robotics and Mechatronics, vol. Vol.28, no. No.2, pp. 194-197, Jan. 2016.

[2] L. ZHANG, Y. GUAN, H. ZHU, and H. ZHANG, "Posture detection of suction modules for a biped wall-climbing robot," Robot, vol. 34, no. 6, p. 758, 2012.

[3] B. Hu, L. Wang, Y. Zhao, and Z. Fu, "A miniature wall climbing robot with biomechanical suction cups," Industrial Robot: An International Journal, vol. 36, no. 6, pp. 551-561, Oct. 2009.

[4] [4] Y. Zhao, Z. Fu, Q. Cao, and Y. Wang, "Development and applications of wall-climbing robots with a single suction cup," Robotica, vol. 22, no. 6, pp. 643-648, Nov. 2004.

[5] Y. Aniroh, "Dynamics And Control of a Suction-Type Wall-Climbing Robot,"IFAC-PapersOnLine, vol. 48, no. 1, pp. 902-903, 2015.

[6] Q. Zhi-yuan, Z. Yan-zheng, F. Zhuang, and W. Y, "Fluid model of sliding suction cup of wall-climbing robots," International Journal of Advanced Robotic Systems, p. 1, 2006.

[7] H. Zhang, J. Zhang, G. Zong, W. Wang, and R. Liu, "Sky cleaner 3: A real pneumatic climbing robot for glass-wall cleaning," IEEE Robotics \& Automation Magazine, vol. 13, no. 1, pp. 32-41, Mar. 2006.

[8] DaijunXu, "Suction Ability Analyses of a Novel Wall Climbing Robot," in 2006 IEEE International Conference on Robotics and Biomimetics, Kunming: IEEE, 2006, pp. 1506-1511.

[9] D. M. Aslam and G. D. Dangi, "Design, fabrication and testing of a smart robotic foot," Robotics and Autonomous Systems, vol. 51, no. 2-3, pp. 207-214, May 2005.

[10] Tohru Miyake, Hidenori Ishihara, and Tatsuya Tomino, "Vacuum-based Wet Adhesion System for Wall Climbing Robots-Lubricating action and seal action by the liquid," in IEEE International Conference on Robotics and Biomimetics, 2008. ROBIO 2008, Bangkok: IEEE, 2009, pp. 1824-1829.

[11] T. C. Apostolescu, C. Udrea, D. Duminica*, G. Ionascu, L. Bogatu, and Laurenţiu Adrian Cartal, "Development of a Climbing Robot with Vacuum Attachment Cups," in Proceedings of International Conference On Innovations, Recent Trends And Challenges In Mechatronics, Mechanical Engineering And New High-Tech Products Development-MECAHITECH'11, 2011, 2011, pp. $258-267$.

[12] Z. Wang, G. Bao, L. Zhang, and Q. Yang, "Development and control of flexible pneumatic wall-climbing robot," Journal of Central South University of Technology, vol. 16, no. 6, pp. 961-970, Dec. 2009.

[13] H. Zhang, W. Wang, J. González-Gómez, and J. Zhang, "Design and realization of a novel modular climbing caterpillar using low- 
frequency vibrating passive suckers," Advanced Robotics, vol. 23, no. 7-8, pp. 889-906, Jan. 2009.

[14] J. Zhu, D. Sun, and S.-K. Tso, "Application of a service climbing robot with motion planning and visual sensing," Journal of Robotic Systems, vol. 20, no. 4, pp. 189-199, Mar. 2003

[15] "(hidden) brand transition Popup," 2016. [Online]. Available: http://www.numatics.com. Accessed: Jun. 19, 2016.

\section{AUTHOR PROFILE}

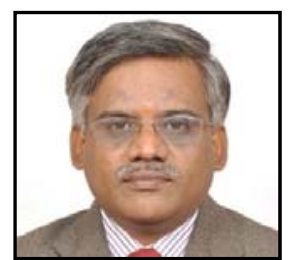

Mr. G Muthukumaran received M.E degree in Computer Science and Engineering from Government College of Technology, Coimbatore and pursuing Ph.D. in Robotics and Control Engineering at Hindustan Institute of Technology and Science, Chennai, India. Currently he is working as Associate Professor in the Dept. of Electronics and Instrumentation Engineering at Hindustan University, Chennai, India. $\mathrm{He}$ is an interdisciplinary and guiding many Research projects at graduate and under graduate level. Earlier he served as Automation Engineer at PSG group of Industries. His research interests are Embedded Systems, Robotics \& Automation.

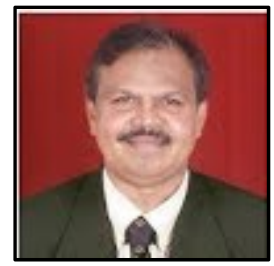

Dr. Uppu Ramachandraiah received M.Tech. degree in Electronics and Instrumentation Engineering from National Institute of Technology, Warangal and Ph.D. degree in Signal Processing from Indian Institute of Technology Madras, India. Currently he is working as Professor\& Head in the Dept. of Electronics and Instrumentation Engineering at Hindustan University, Chennai, India. He is an interdisciplinary and guiding 9 Research scholars. Earlier he served as Senior Scientific Officer at Bhabha Atomic Research Centre Facilities, Department of Atomic Energy. His research interests are Real-time Embedded Systems, Real-time Signal Processing, Robotics and Automation and Industrial Automation. 